\title{
Eficiência da aplicação da mistura de glyphosate com saflufenacil sobre
}

\section{plantas de Brachiaria decumbens ${ }^{1}$}

\section{Efficiency of the application of the mixture of glyphosate with saflufenacil on \\ Brachiaria decumbens plants}

\author{
Juliana Roberta Gobi Queiroz²; Antonio Carlos da Silva Junior ${ }^{3}$; Andréia Cristina Peres \\ Rodrigues da Costa ${ }^{4}$; Dagoberto Martins ${ }^{5}$
}

\begin{abstract}
Resumo - O objetivo deste estudo foi verificar o sinergismo ou antagonismo de glyphosate com e sem a mistura de saflufenacil no controle de $B$. decumbens. Foram avaliados no estudo os seguintes tratamentos: glyphosate (1.080), glyphosate + saflufenacil + Dash $(1.080+24,5+0,5 \%$ v/v), glyphosate + 2,4 D (1.080 + 720); glyphosate (1.440); glyphosate + saflufenacil + Dash (1.440 + 24,5 + 0,5\% v/v); glyphosate + 2,4 D (1.440 + 720); saflufenacil + Dash $(24,5+0,5 \mathrm{v} / \mathrm{v}) ; 2,4 \mathrm{D}$ (720), doses em g i.a./e.a. ha ${ }^{-1}$. A eficiência de controle de $B$. decumbens foi avaliada em diversos períodos: $3,7,14,21,28,35,42$ e 49 dias após a aplicação (DAA), com base nos sintomas de fitointoxicação, utilizando-se a escala de $0 \%$ a $100 \%$. Os resultados foram submetidos à análise de variância pelo teste $\mathrm{F}$ e as médias comparadas pelo teste Tukey ao nível de 5\% de probabilidade. $\mathrm{O}$ herbicida glyphosate aplicado de forma isolada nas duas doses testadas, ou em mistura com saflufenacil, proporcionou um excelente controle das plantas de B. decumbens já a partir dos 14 dias. Os herbicidas saflufenacil e 2,4-D quando aplicados isoladamente sem a presença do herbicida glyphosate mostraram-se ineficientes no controle das plantas. O controle das plantas de braquiária proporcionado pelas misturas de glyphosate com 2,4-D e saflufenacil foi semelhante ao da aplicação isolada de glyphosate. As misturas não apresentaram sinergismo ou antagonismo sobre o controle das plantas de $B$. decumbens.
\end{abstract}

Palavras-chaves: planta daninha, herbicida, sinergismo, antagonismo

Abstract - The aim of this work was to investigate the synergism or antagonism of glyphosate with and without mixing with saflufenacil to control $B$. decumbens. The following treatments were evaluated: glyphosate (1.080); glyphosate + saflufenacil + Dash $(1,080+24.5+0.5 \% \mathrm{v} / \mathrm{v})$; glyphosate + 2,4-D (1,080 + 720); glyphosate $(1,440)$; glyphosate + saflufenacil + Dash $(1,440+$ $24.5+0.5 \% \mathrm{v} / \mathrm{v}) ;$ glyphosate + 2,4-D $(1,440+720)$; saflufenacil + Dash $(24.5+0.5 \mathrm{v} / \mathrm{v}) ; 2,4-\mathrm{D}$ (720), doses in $\mathrm{g}$ a.i./e.a. $\mathrm{ha}^{-1}$. The efficiency control of Brachiaria decumbens was evaluated at different times: 3, 7, 14, 21, 28, 35, 42 and 49 days after application (DAA), with basis on

\footnotetext{
${ }^{1}$ Recebido para publicação em 08/04/2014 e aceito em 05/05/2015.

${ }^{2}$ Aluna de Doutorado do PPG em Agricultura - FCA/UNESP. Botucatu, SP, Brasil. jugobiagro@ gmail.com (*autor para correspondência)

3 Aluno de Doutorado do PPG em Produção Vegetal - FCAV/UNESP. Jaboticabal, SP, Brasil. acsjr_agro@hotmail.com

${ }^{4}$ Professora Adjunto do Departamento de Ciências Agronômicas - UEM. Umuarama, PR, Brasil. acprcosta@uem.br

5 Professor Livre Docente do Departamento de Produção Vegetal - FCAV/UNESP. Jaboticabal, SP, Brasil. dmartins@fcav.unesp.br
} 
phytotoxicity symptoms, using the scale of $0 \%$ to $100 \%$. The results were submitted to the F test and the average values were compared using Tukey test at $5 \%$ probability. The herbicide glyphosate applied isolated at both tested doses, or mixed with saflufenacil, provided excellent control of $B$. decumbens after 14 days. Saflufenacil and 2,4-D when applied isolated without the presence of glyphosate were ineffective in controlling plants. The control of $B$. decumbens provided by mixtures of glyphosate with 2,4-D and saflufenacil was similar to the application of glyphosate isolated. Mixtures showed no synergism or antagonism on the control of $B$. decumbens plants.

Keywords: weed, herbicide, synergism, antagonism

\section{Introdução}

Os diversos cultivares de capimbraquiária que foram introduzidos no Brasil são originários da África do Sul. Trata-se de uma excelente forrageira, perene e com grande produção de massa foliar de boa qualidade, resistente ao pastejo e ao pisoteio e que protege o solo contra erosão. Em áreas onde a espécie foi introduzida como forrageira, ao transformar essas terras em lavouras, o capim-braquiária passa a constituir-se em uma importante planta infestante, muito agressiva e de difícil controle. Devido ao caráter exótico e elevada agressividade, essas plantas passaram a invadir áreas de agricultura, fruticultura e reflorestamento, especialmente a espécie Brachiaria decumbens Stapf. (Bianco et al., 2005).

A dificuldade de controle das braquiárias tem sido atribuída principalmente às características morfológicas das plantas, à grande quantidade de sementes no solo, que forma um banco de sementes e, eventualmente, à presença em algumas espécies de Brachiaria de compostos alelopáticos que inibem o crescimento de outras forrageiras (Rodrigues; Reis, 1994). Existem diferentes métodos para o controle das plantas daninhas. O controle químico é o que tem sido mais utilizado, principalmente devido às extensas áreas cultivadas. No plantio direto, o controle das plantas daninhas depende da utilização de herbicidas, uma vez que os cultivos e as capinas são incompatíveis com a tecnologia utilizada no sistema. O manejo de plantas daninhas ou dessecação que antecede o plantio direto, é essencial para um bom desenvolvimento das culturas. A eliminação de plantas daninhas antes da semeadura permite que a cultura tenha um desenvolvimento inicial rápido e vigoroso.

Como as espécies de plantas infestantes apresentam-se, quase sempre, de maneira muito heterogênea no solo, a utilização simultânea ou seqüencial de dois ou mais herbicidas vem sendo cada vez mais empregada na agricultura e representa um avanço nas estratégias de controle sobre as plantas daninhas (Gressel, 1990). Existe grande número de fatores que podem interferir nas interações entre as misturas de herbicidas (Sorensen et al., 1987).

$\mathrm{O}$ herbicida saflufenacil pertence à família dos pirimidinedione, e inibe a enzima oxidase protoporfirinogênio - IX. Este herbicida possui uma atividade de contato sobre plantas daninhas dicotiledôneas. Quando em mistura com o herbicida glyphosate em préplantio (manejo total da área), melhora o controle quando comparado com a ação do glyphosate isolado, além de fornecer um bom controle residual (Anonymous, 2008).

Segundo Colby (1967), quando a resposta da mistura de herbicidas é maior que a esperada, a mistura é sinergística; quando é menor que a esperada, é antagonística e quando igual, aditiva. Despertam particular interesse as misturas que apresentam sinergismo, pois permitem o uso de doses menores e controlam plantas daninhas resistentes (Gressel, 1990). Esse sinergismo, muitas vezes, ocorre quando são misturados dois herbicidas que apresentam diferentes mecanismos de ação, nos quais pode haver uma ação de complementaridade entre os 
mesmos, com um facilitando a ação física e ou bioquímica do outro (Matthews, 1994).

Dessa forma a mistura de herbicidas no tanque de pulverização pode oferecer alguns benefícios, quando comparado com o uso de apenas um produto, como aumento do número de espécies controladas, melhor controle de determinadas espécies, redução do custo de pulverização e possibilidade de redução da dose recomendada, consequentemente a redução de resíduos no solo e da toxicidade à cultura (Machado et al., 2006).

Considerando-se a importância do controle dessa planta daninha, principalmente em cultivos onde não há o revolvimento do solo, o presente trabalho teve por objetivo estudar o sinergismo ou antagonismo de glyphosate com e sem a mistura de saflufenacil no controle de $B$. decumbens.

\section{Material e Métodos}

O estudo foi conduzido na Faculdade de Ciências Agronômicas/UNESP, Campus de
Botucatu/SP, utilizando-se um solo classificado como Latossolo Vermelho-escuro textura média, Unidade Patrulha. Sementes de Brachiaria decumbens foram semeadas em vasos com capacidade de 5,0 L, contendo solo e fertilizante. A aplicação dos tratamentos foi realizada quando as plantas de $B$. decumbens apresentavam-se no estádio fenológico de emborrachamento/pré-florescimento.

Os tratamentos foram dispostos em delineamento inteiramente casualizado, com quatro repetições (Tabela 1). Cada parcela experimental foi constituída de um vaso contendo uma planta de $B$. decumbens.

A aplicação foi realizada com um pulverizador estacionário pressurizado a ar comprimido, com pressão constante de $200 \mathrm{kPa}$, equipado com barra de pulverização munida de quatro pontas de jato plano tipo Teejet XR $11002 \mathrm{VS}$, com espaçamento de $0,5 \mathrm{~m}$ entre os bicos. A barra de pulverização foi deslocada na velocidade de $1 \mathrm{~m} \mathrm{~s}^{-1}$, o que proporcionou um consumo de calda de 200 L.ha $^{-1}$.

Tabela 1. Doses e herbicidas testados no controle de plantas de Brachiaria decumbens. Botucatu, SP.

\begin{tabular}{lc}
\hline \multicolumn{1}{c}{ Tratamentos } & Dose $\left(\mathrm{g}\right.$ i.a./e.a. ha $\left.{ }^{-1}\right)$ \\
\hline Testemunha & - \\
Glyphosate & 1.080 \\
Glyphosate + saflufenacil + Dash & $1.080+24,5+0,5 \% \mathrm{v} / \mathrm{v}$ \\
Glyphosate + 2,4-D & $1.080+720$ \\
Glyphosate & 1.440 \\
Glyphosate + saflufenacil + Dash & $1.440+24,5+0,5 \% \mathrm{v} / \mathrm{v}$ \\
Glyphosate + 2,4-D & $1.440+720$ \\
Saflufenacil + Dash & $24,5+0,5 \% \mathrm{v} / \mathrm{v}$ \\
2,4-D & 720 \\
\hline
\end{tabular}
glyphosate: Roundup WG $\left(720 \mathrm{~g} \mathrm{Kg}^{-1}\right)$

A eficiência de controle de $B$. comparadas pelo teste Tukey ao nível de $5 \%$ de decumbens foi avaliada em diversos períodos: 3 , probabilidade. A análise de variância e 7, 14, 21, 28, 35, 42 e 49 dias após a aplicação (DAA), com base nos sintomas de comparação de médias foi feita pelo programa SISVAR. fitointoxicação, utilizando-se a escala de $0 \%$ a $100 \%$, onde $0 \%$ representa ausência total de injúria e $100 \%$ a morte das plantas (SBCPD, 1995).

Os resultados foram submetidos à análise de variância pelo teste $\mathrm{F}$ e as médias 


\section{Resultados e Discussão}

Na Tabela 2 estão apresentados os valores médios de controle das plantas de $B$. decumbens em função da aplicação de herbicidas isolados ou em misturas. Observouse já em 3 DAA dos herbicidas que as plantas que foram submetidas aos tratamentos com o herbicida saflufenacil na forma isolada ou em mistura com o herbicida glyphosate nas doses de 1.080 e 1.440 g e.a. ha ${ }^{-1}$ apresentaram os primeiros sintomas de fitotointoxicação, porém ainda com um controle incipiente. O controle inicial proporcionado pelo herbicida saflufenacil aplicado isolado decresceu a partir de 7 DAA e aos 14 DAA esses sintomas de injúrias quase não eram observados.

Tabela 2. Valores médios de controle de plantas de Brachiaria decumbens em função da aplicação de herbicidas. Botucatu, SP.

\begin{tabular}{|c|c|c|c|c|c|c|c|c|c|}
\hline \multirow{2}{*}{ Herbicidas } & \multirow{2}{*}{$\begin{array}{c}\text { Dose } \\
\left(\mathrm{g} \text { i.a/e.a ha }{ }^{-1}\right)\end{array}$} & \multicolumn{8}{|c|}{ \% controle - Dias após a aplicação } \\
\hline & & 3 & 7 & 14 & 21 & 28 & 35 & 42 & 49 \\
\hline Testemunha & - & $0,0 \mathrm{~b}$ & $0,0 \mathrm{~d}$ & $0,0 \mathrm{~b}$ & $0,0 \mathrm{~b}$ & $0,0 \mathrm{~b}$ & $0,0 \mathrm{~b}$ & $0,0 \mathrm{~b}$ & $0,0 \mathrm{~b}$ \\
\hline Glyphosate & 1.080 & $0,3 \mathrm{~b}$ & $77,5 \mathrm{a}$ & $100 \mathrm{a}$ & $100 \mathrm{a}$ & $100 \mathrm{a}$ & $100 \mathrm{a}$ & $100 \mathrm{a}$ & $100 \mathrm{a}$ \\
\hline Glyphosate + saf. .1 & $1.080+24,5$ & $14,3 \mathrm{a}$ & $60,0 \mathrm{ab}$ & 99,3 a & $99,5 \mathrm{a}$ & $99,5 \mathrm{a}$ & $99,5 \mathrm{a}$ & $99,8 \mathrm{a}$ & $99,8 \mathrm{a}$ \\
\hline Glyphosate + 2,4-D & $1.080+720$ & $1,0 \mathrm{~b}$ & $14,3 \mathrm{~cd}$ & $96,5 \mathrm{a}$ & $98,5 \mathrm{a}$ & $99,3 \mathrm{a}$ & $99,5 \mathrm{a}$ & $99,8 \mathrm{a}$ & $99,8 \mathrm{a}$ \\
\hline Glyphosate & 1.440 & $1,3 \mathrm{~b}$ & $58,8 \mathrm{ab}$ & $100 \mathrm{a}$ & $100 \mathrm{a}$ & $100 \mathrm{a}$ & $100 \mathrm{a}$ & $100 \mathrm{a}$ & $100 \mathrm{a}$ \\
\hline Glyphosate + saf. .1 & $1.440+24,5$ & $13,3 \mathrm{a}$ & $68,8 \mathrm{a}$ & $95,0 \mathrm{a}$ & $99,3 \mathrm{a}$ & $99,8 \mathrm{a}$ & $100 \mathrm{a}$ & $100 \mathrm{a}$ & $100 \mathrm{a}$ \\
\hline Glyphosate + 2,4-D & $1.440+720$ & $0,8 \mathrm{~b}$ & $38,3 \mathrm{bc}$ & $100 \mathrm{a}$ & $100 \mathrm{a}$ & $100 \mathrm{a}$ & $100 \mathrm{a}$ & $100 \mathrm{a}$ & $100 \mathrm{a}$ \\
\hline Saflufenacil ${ }^{11}$ & 24,5 & $13,0 \mathrm{a}$ & $8,5 \mathrm{~d}$ & $1,5 \mathrm{~b}$ & $0,0 \mathrm{~b}$ & $0,0 \mathrm{~b}$ & $0,0 \mathrm{~b}$ & $0,0 \mathrm{~b}$ & $0,0 \mathrm{~b}$ \\
\hline 2,4-D & 720 & $0,3 \mathrm{~b}$ & $0,0 \mathrm{~d}$ & $0,0 \mathrm{~b}$ & $0,0 \mathrm{~b}$ & $0,0 \mathrm{~b}$ & $0,0 \mathrm{~b}$ & $0,0 \mathrm{~b}$ & $0,0 \mathrm{~b}$ \\
\hline$F_{\text {Herbicid }}$ & & $32,3^{*}$ & $26,5^{*}$ & $761^{*}$ & $8139^{*}$ & $9701^{*}$ & $4850^{*}$ & $9700^{*}$ & $9700^{*}$ \\
\hline $\mathrm{CV}(\%)$ & & 46,66 & 33,33 & 5,40 & 1,11 & 0,61 & 0,71 & 0,35 & 0,35 \\
\hline d.m.s. & & 5,429 & 28,730 & 8,455 & 1,759 & 0,972 & 1,122 & 0,561 & 0,561 \\
\hline
\end{tabular}

${ }^{11}$ Adicionou-se Dash a 0,5\% v/v. glyphosate: Roundup WG saf.- saflufenacil. * Significativo a 5\% de probabilidade. Médias seguidas da mesma letra não diferem estatisticamente entre si pelo teste de Tukey $(\mathrm{P}>0,05)$.

Aos 7 DAA os melhores desempenhos de controle foram observados para o tratamento com glyphosate nas doses de 1.080 e $1.440 \mathrm{~g}$ e.a. $\mathrm{ha}^{-1}$ aplicado isolado e em mistura com o herbicida saflufenacil, com controle variando entre 58,8 e 77,5\%. Esses efeitos de fitointoxicação, em virtude do mecanismo de ação do glyphosate, são perceptíveis apenas em alguns dias, dependendo das condições ambientais (Rodrigues; Almeida, 1998).

A aplicação isolada do herbicida glyphosate nas duas doses (1.080 e 1.440 g e.a. $\left.\mathrm{ha}^{-1}\right)$ e a sua mistura com os herbicidas saflufenacil e 2,4-D praticamente promoveu o controle total de $B$. decumbens aos 14 DAA. Ressalta-se que o excelente controle observado se estendeu até 49 DAA, com pequena rebrota nas parcelas tratadas a dose de $1.080 \mathrm{~g}$ e.a. ha $^{-1}$ de glyphosate.
Para a aplicação isolada de glyphosate, Timossi et al. (2006), estudando o uso desse herbicida no manejo químico de espécies de braquiária, observaram que o controle tanto da $B$. decumbens quanto da $B$. brizantha foi satisfatório a partir da dosagem de $2.160 \mathrm{~g}$ e.a. ha $^{-1}$ de glyphosate, contudo, também não foi capaz de evitar totalmente a probabilidade de rebrotes. Nunes et al. (2010) observaram que o controle com glyphosate, nas doses de 1.440 e 2.160 g e.a. ha ${ }^{-1}$ foi satisfatório aos 10 e 20 dias após a aplicação nas espécies $B$. decumbens e $B$. brizantha. Pereira (1996) também observou que a dosagem de $1.440 \mathrm{~g}$ e.a. ha $^{-1}$ foi eficaz no controle de $B$. decumbens. De acordo com Brighenti et al. (2011) aos 21 DAA, o herbicida glyphosate na dose de 1.080 g e.a. ha ${ }^{-1}$ apresentou percentagem de controle próximo a $75 \%$ para essa mesma espécie. Essas diferenças de eficácia nas dosagens desse herbicida podem 
ser explicadas também pela diferença de idade das plantas no momento do manejo.

A mistura de glyphosate nas duas doses testadas com o herbicida saflufenacil, não interferiu nos resultados quando comparados com aplicação isolada do herbicida glyphosate (Tabela 2), neste caso, demonstrando não haver nenhum efeito negativo na associação destes dois herbicidas. Os herbicidas saflufenacil e 2,4D mostraram-se ineficientes no controle das plantas de $B$. decumbens quando aplicados isolados.

Guerra et al. (2012) ao avaliarem a eficácia de alguns herbicidas e misturas destes no controle de $B$. plantaginea, também observaram que todos os tratamentos foram eficientes no controle dessa espécie e que não houve antagonismo na mistura saflufenacil e glyphosate, entretanto pelo controle aos 40 DAA já se faz necessário uma aplicação em pósemergência de glyphosate ou então de graminicidas específicos para este fim.

Quando foi aplicada a mistura de glyphosate + 2,4-D, notou-se aos 7 DAA que o 2,4-D prejudicou a ação do glyphosate sobre as plantas de B. decumbens. Já aos 14 DAA, este efeito dissipou-se resultando em um excelente controle das plantas de braquiária (Tabela 2). A literatura relata efeito prejudicial do 2,4-D sobre ação graminicida de herbicidas como o glyphosate. Os efeitos antagônicos ocorrem principalmente quando o graminicida é aplicado juntamente ou após os herbicidas latifolicidas (Trezzi et al., 2007). Rockenbach et al. (2012) em estudo com plantas de azevém, observaram que o glyphosate aplicado de forma isolada, nas duas doses testadas (720 e 1.440 g e.a. ha ${ }^{-1}$ ), foi eficaz no controle dessas plantas, entretanto, nas três épocas avaliadas (14, 28 e 42 DAA), quando foi adicionado o 2,4-D (670 e $1.005 \mathrm{~g}$ e.a. ha $\left.{ }^{-1}\right)$, houve redução do nível de controle de azevém na menor dose do glyphosate. Todavia, o aumento da dose de glyphosate para $1.440 \mathrm{~g}$ e.a. ha ${ }^{-1}$ superou esse prejuízo a partir de 28 dias após a aplicação (Rockenbach et al., 2012).
Tonietti et al. (2012) também estudaram a interferência das associações de diferentes doses de glyphosate e 2,4-D sobre o controle de $B$. decumbens. Estes pesquisadores observaram que a aplicação isolada de glyphosate nas dosagens 1.920 e 2.400 g i.a. ha ${ }^{-1}$ atingiram $100 \%$ de controle dessa espécie, e quando associaram dose de 2.400 g i.a. ha ${ }^{-1}$ de glyphosate com 2.418 g i.a. ha ${ }^{-1}$ de 2,4-D ocorreu uma redução de controle de $22 \%$, não atingindo o mínimo aceitável de $80 \%$, resultando na hipótese de ocorrência de antagonismo entre os dois ingredientes ativos. $\mathrm{O}$ aumento da dose do herbicida 2,4-D quando aplicado associado ao glyphosate no controle dessa braquiária resultou em perdas de eficiência do glyphosate.

Costa et al. (2012) observaram que o herbicida glyphosate aplicado isolado na dose 1.440 g i.a. ha ${ }^{-1}$ promoveu controle de $80 \%$ para a B. decumbens e a dose $2.400 \mathrm{~g}$ i.a ha ${ }^{-1}$ promoveu controle total. As doses de 806 e 1.612 g i.a. ha ${ }^{-1}$ de 2,4-D não interferiram na redução da eficiência do glyphosate $(960,1.440$ e $2.400 \mathrm{~g}$ i.a. ha ${ }^{-1}$ ) no controle dessa espécie, quando utilizados de forma associada. Já o 2,4D nas doses de 2.418 e 3.224 g i.a. ha ${ }^{-1}$ associado ao glyphosate $\left(960,1.440\right.$ e 2.400 g i.a. ha $\left.{ }^{-1}\right)$, promoveu perda de eficiência do glyphosate no controle da B. decumbens, reduzindo o controle abaixo do mínimo aceitável de $80 \%$.

\section{Conclusões}

O herbicida glyphosate aplicado de forma isolada nas duas doses testadas, ou em mistura com saflufenacil proporcionou um excelente controle das plantas de Brachiaria decumbens já a partir dos 14 dias. Os herbicidas saflufenacil e 2,4-D quando aplicados isoladamente sem a presença do herbicida glyphosate mostraram-se ineficientes no controle das plantas.

O controle das plantas de braquiária proporcionado pelas misturas de glyphosate com 2,4D e saflufenacil foi semelhante ao da aplicação isolada de glyphosate. As misturas 
não apresentaram sinergismo ou antagonismo no controle das plantas de $B$. decumbens.

\section{Referências}

ANONYMOUS. Kixor herbicide word dwide technical brochure. Germany, BASF Agricultural Products, Research Triangle Park, NC 27709, 18 p., 2008.

BIANCO, S.; TONHAO, M.A.R.; PITELLI, R.A. Crescimento e nutrição mineral de capimbraquiária. Planta Daninha, v.23, n.3, p. 423428, 2005.

BRIGHENTI, A.M. et al. Suscetibilidade diferencial de espécies de braquiária ao herbicida glifosato. Pesquisa Agropecuária Brasileira, v.46, n.10, p.1241-1246, 2011.

COLBY, S.R. Calculating synergistic and antagonistic responses of herbicide combinations. Weeds, v.15, p.20-22, 1967.

COSTA L.H. et al. Influência do herbicida 2,4d na eficácia do herbicida glyphosate no controle de diferentes espécies de plantas daninhas. In: CONGRESSO BRASILEIRO DA CIÊNCIA DAS PLANTAS DANINHAS, 28. 2012, Campo Grande. Resumos... SBCPD, 2012. p.162.

GRESSEL, J. Synergizing herbicides. Reviews of Weed Science, v.5, p.49-82, 1990.

GUERRA, D.G. et al. Utilização de saflufenacil em aplicação pré-semeadura na cultura da soja. In: CONGRESSO BRASILEIRO DA CIÊNCIA DAS PLANTAS DANINHAS, 28, 2012, Campo Grande. Resumos... SBCPD, 2012. p. 275.

MACHADO, A.F.L. et al. Misturas de herbicidas no manejo de plantas daninhas na cultura do feijão. Planta Daninha, v.24, n.1, p.107-114, 2006.

MATTHEWS, J.M. Management of herbicide resistant weed populations. In: POWLES, S.; HOLTUM, J. (eds.) Herbicide resistance in plants: biology and biochemistry. Boca Raton: CRC, 1994. p.317-335.

NUNES, A.S. et al. Formação de cobertura vegetal e manejo de plantas daninhas na cultura da soja em sistema plantio direto. Planta Daninha, v.28, n.4, p.727-733, 2010.

PEREIRA, F.A.R. Determinação da dose eficiente de dessecantes sistêmicos no manejo de Brachiaria decumbens em plantio direto da soja, na região dos cerrados. In: SEMINÁRIO: ZAPP - O DESAFIO DO NOVO, 1995, São Paulo. Resumos... Zeneca Agrícola, 1996. p.9596.

ROCKENBACH, A.P.; SCHNEIDER, T.; BIANCHI, M.A. Associação de 2,4-D com glyphosate e com inibidores da accase no controle de azevém (Lolium multiflorum). In: CONGRESSO BRASILEIRO DA CIÊNCIA DAS PLANTAS DANINHAS, 28. 2012, Campo Grande. Resumos... SBCPD, 2012. p.263.

RODRIGUES, B.N.; ALMEIDA, F.S. Guia de herbicidas: contribuição para uso adequado em plantio direto. 4.ed. Londrina: IAPAR, 1998. 648p.

RODRIGUES, L.R.A.; REIS, R.A. Estabelecimento de outras forrageiras em áreas de Brachiaria spp. In: SIMPÓSIO SOBRE MANEJO DA PASTAGEM, 11. 1994, Piracicaba. Resumos... FEALQ, 1994. p.299325.

SOCIEDADE BRASILEIRA DA CIÊNCIA DAS PLANTAS DANINHAS. Procedimentos para instalação, avaliação e análise de experimentos com herbicidas. Londrina: SBCPD, 1995. 42p.

SORENSEN, V.M.; MEGGITT, W.F.; PENNER, D. The interaction of acifluorfen and bentazon in herbicidal combinations. Weed Science, v.35, n.4, p.449-456, 1987.

TREZZI, M.M. et al. Antagonismo das associações de clodinafop-propargyl com metsulfuronmethyl e 2,4-D no controle de 
azevém (Lolium multiflorum). Planta Daninha, v.25, n.4, p.839-847, 2007.

TIMOSSI, P.C.; DURIGAN, J.C.; LEITE, G.J. Eficácia de glifosato em plantas de cobertura. Planta Daninha, v.24, n.2, p.475-480, 2006.

TONIETTI, A.N. et al. Interferência do herbicida 2,4-D sobre a eficácia do herbicida glifosato no controle de capim-braquiária. In: CONGRESSO BRASILEIRO DA CIÊNCIA DAS PLANTAS DANINHAS, 28. 2012, Campo Grande. Resumos... SBCPD, 2012. p. 176. 